\title{
Ce:YAG transparent ceramics based on nanopowders produced by laser ablation method: Fabrication, optical and scintillation properties
}

\author{
V. V. Osipov ${ }^{1}$, V. A. Shitov ${ }^{1}$, K. E. Lukyashin ${ }^{1 *}$, R. N. Maksimov ${ }^{1,2}$, A. V. Ishchenko ${ }^{2}$, V. V. Platonov ${ }^{1}$ \\ ${ }^{1}$ Institute of Electrophysics UrB RAS, Amundsena St. 106, Ekaterinburg 620016, Russia \\ ${ }^{2}$ Ural Federal University, Mira St. 19, Ekaterinburg 620002, Russia
}

*kostya@iep.uran.ru

DOI 10.17586/2220-8054-2017-8-3-351-359

\begin{abstract}
Transparent Ce:YAG ceramics were fabricated by the solid-state reaction method with an additional round of pre-calcining using nanopowders of $\mathrm{Ce}_{2 x} \mathrm{Y}_{2-2 x} \mathrm{O}_{3}(x=0.001,0.01,0.03$ and 0.05$)$ and $\mathrm{Al}_{2} \mathrm{O}_{3}$ synthesized by laser ablation. Additional pre-calcining of the nanopowder mixture in air was used to partially transform the oxides into the YAG phase before sintering. The transmittance of the obtained 2-mm-thick Ce:YAG ceramics was over $81 \%$ in the wavelength range of 500-900 nm. The average volumes of the scattering centers in the obtained ceramics were evaluated by direct count method to be 34, 74, 53, $50 \mathrm{ppm}$ for $0.1,1,3$ and $5 \%$ Ce doping concentration, respectively. The absolute light yields, energy resolutions under $662 \mathrm{keV}$ gamma ray, and decay curves of scintillations of the fabricated Ce:YAG samples were measured and compared to those of Ce:YAG and CsI:Tl single crystals.
\end{abstract}

Keywords: Laser ablation, nanopowder, yttrium aluminium garnet, cerium, scintillator, transparent ceramics, transmittance.

Received: 14 February 2017

Revised: 20 March 2017

\section{Introduction}

Most of the scintillation materials used in ionizing radiation detectors have been obtained in a single crystalline form. It is well known that growing high melting point scintillation crystals is time-consuming and expensive. For this application, one of the most promising approaches is to replace single crystals by ceramic materials. For example, to date, several scintillation ceramics have been successfully used in medical imaging devices (General Electric's premium X-ray CT) $[1,2]$.

The important factors that influence the scintillation properties include a high optical quality of material, effective atomic number, decay time of scintillations, and uniformity distribution of activator ions in the host lattice. Growing high-quality large-sized single crystals with a homogeneous dopant distribution is difficult due to doping gradients in the melt. Ceramic technology offers the possibility for fabricating highly transparent and large-sized garnet materials at lower temperatures and with a homogeneous or controlled gradient activator ion distribution $[3,4]$.

One of the most famous and most modern ceramic scintillators is a well-known scintillator based on $\mathrm{Ce}^{3+}$. doped $\mathrm{Y}_{3} \mathrm{Al}_{5} \mathrm{O}_{12}(\mathrm{Ce}: \mathrm{YAG})$ with a garnet structure [5]. YAG is a promising candidate for scintillator applications because of its excellent optical and thermomechanical properties, high electron conversion efficiency, good energy resolution, reasonably fast decay time, mechanical ruggedness and durability, good thermal conductivity, and vacuum compatibility [6]. Ce-doped YAG single crystals and ceramic materials are widely used in medical imaging devices [1,7], white LED applications [8-10], white high-power LEDs [11], electron microscopes, $\beta$-, soft $\gamma$ - or X-ray counters and spectrometers, and electron- or X-ray image screens [12].

Additionally, polycrystalline laser ceramics are extremely attractive materials due to their ease of fabrication, low cost, unlimited dimensions, high doping concentration of activator ions and multifunctional composite structure. To fabricate highly transparent ceramics, it is necessary to obtain thin grain boundaries $(\sim 1 \mathrm{~nm})$ and a pore-free structure. There are two main methods for meeting these requirements. In the first approach, referred to as the solid state reaction method (SSR) [13] or the reactive sintering method [14], commercial oxide powders are mixed together, then the mixture is dried, pressed and sintered to transparency. Thus, the phase transformations of $\mathrm{Y}_{2} \mathrm{O}_{3}$ and $\mathrm{Al}_{2} \mathrm{O}_{3}$ into $\mathrm{Y}_{3} \mathrm{Al}_{5} \mathrm{O}_{12}$ occur at the vacuum sintering.

According to the second approach $[15,16]$, ceramics are synthesized from YAG nanopowder. Chemical coprecipitation route $[15,16]$, citrate gel [17] and gel combustion methods $[18,19]$ are used to produce this type of nanopowder.

However, the optical quality of YAG-based ceramics obtained from nanopowders produced by the laser ablation method is better when phase transformation into YAG partially occurs before vacuum sintering [20]. 
In this paper, we fabricate transparent Ce:YAG ceramic samples with different $\mathrm{Ce}^{3+}$ concentrations and report on the microstructural, optical, radioluminescence and scintillation properties of the obtained ceramics. We believe that the synthesis of nanopowders for transparent ceramics is a key parameter to simultaneously achieve a high doping level and uniform activator ion distribution in bulk ceramic scintillators. In this regard, a laser ablation technique used for the synthesis of raw materials is a very attractive method to produce particles with a complex chemical composition because the required components could be properly and homogeneously mixed at the atomic scale in vapors during the process of evaporation and condensation.

\section{Experimental}

The pulse-periodic $\mathrm{CO}_{2}$ laser LAERT $(\lambda=10.6 \mu \mathrm{m})$ [21] was used to produce nanopowders. The main laser parameters at the synthesis of nanopowders were as follows: average power $P=600-800 \mathrm{~W}$, peak power $P_{p}=10-11 \mathrm{~kW}$, half-height duration $\tau_{l}=200 \mu \mathrm{s}$, pulse repetition rate $f=500 \mathrm{~Hz}$.

In the present study, coarse $\alpha-\mathrm{Al}_{2} \mathrm{O}_{3}\left(>99.99 \%, 150 \mathrm{~nm}\right.$, Sumitomo chemical Co., Ltd., Tokyo, Japan), $\mathrm{Y}_{2} \mathrm{O}_{3}$ (> $99.99 \%, 1-20 \mu \mathrm{m}$, Lanhit, Moscow, Russia) and $\mathrm{Nd}_{2} \mathrm{O}_{3}(>99.99 \%, 1-15 \mu \mathrm{m}$, Lanhit, Moscow, Russia) were used as initial powders. Yttrium and neodymium oxides were mixed together for $24 \mathrm{~h}$ in a rotary mixer with inclined axis of rotation to prepare $\mathrm{Ce}_{2 x} \mathrm{Y}_{2-2 x} \mathrm{O}_{3}(x=0.001,0.01,0.03$, and 0.05) laser target. Furthermore, the mixture of coarse powders (or $\mathrm{Al}_{2} \mathrm{O}_{3}$ powder) was compacted with a uniaxial static press at the pressure of $10 \mathrm{MPa}$. Subsequently, the targets were sintered at $1300{ }^{\circ} \mathrm{C}$ for $5 \mathrm{~h}$ in air so that prepared targets would not be destroyed under the action of laser radiation during the synthesis of nanopowders. After sintering, the laser targets were $60 \mathrm{~mm}$ in diameter and $19 \mathrm{~mm}$ in thickness.

The $\mathrm{Ce}^{3+}: \mathrm{Y}_{2} \mathrm{O}_{3}$ nanopowder was produced at evaporation of $0.1,1,3,5$ at. $\% \mathrm{Ce}^{3+}: \mathrm{Y}_{2} \mathrm{O}_{3}$ target material with eventual condensation of vapor in air flow. Particles are weakly agglomerated and have a weakly faceted nearspherical shape. Fig. 1(c) shows the size distribution of $\mathrm{Ce}^{3+}: \mathrm{Y}_{2} \mathrm{O}_{3}$ nanopowder (Fig. 1(a)) after sedimentation. Sedimentation of nanopowder after laser evaporation was used for removal of splinters of a target and drops of material. The distribution peak is realized at $13 \mathrm{~nm}$, the average particle size being $13 \mathrm{~nm}$. According to the BET analysis (TriStar 3000), the specific surface area of the $\mathrm{Ce}^{3+}: \mathrm{Y}_{2} \mathrm{O}_{3}$ particles was $51.23 \mathrm{~m}^{2} / \mathrm{g}$.

$\mathrm{X}$-ray diffraction analysis (XRD) on D8 Discover diffractometer has shown that the particles of the $\mathrm{Ce}^{3+}: \mathrm{Y}_{2} \mathrm{O}_{3}$ nanopowder have a monoclinic $\gamma-\mathrm{Y}_{2} \mathrm{O}_{3}$ phase. The transformation of metastable monoclinic phase in a main cubic is accompanied by substantial growth of elementary cell volume ( $a=12.011 \AA$ ). Therefore, in order to avoid
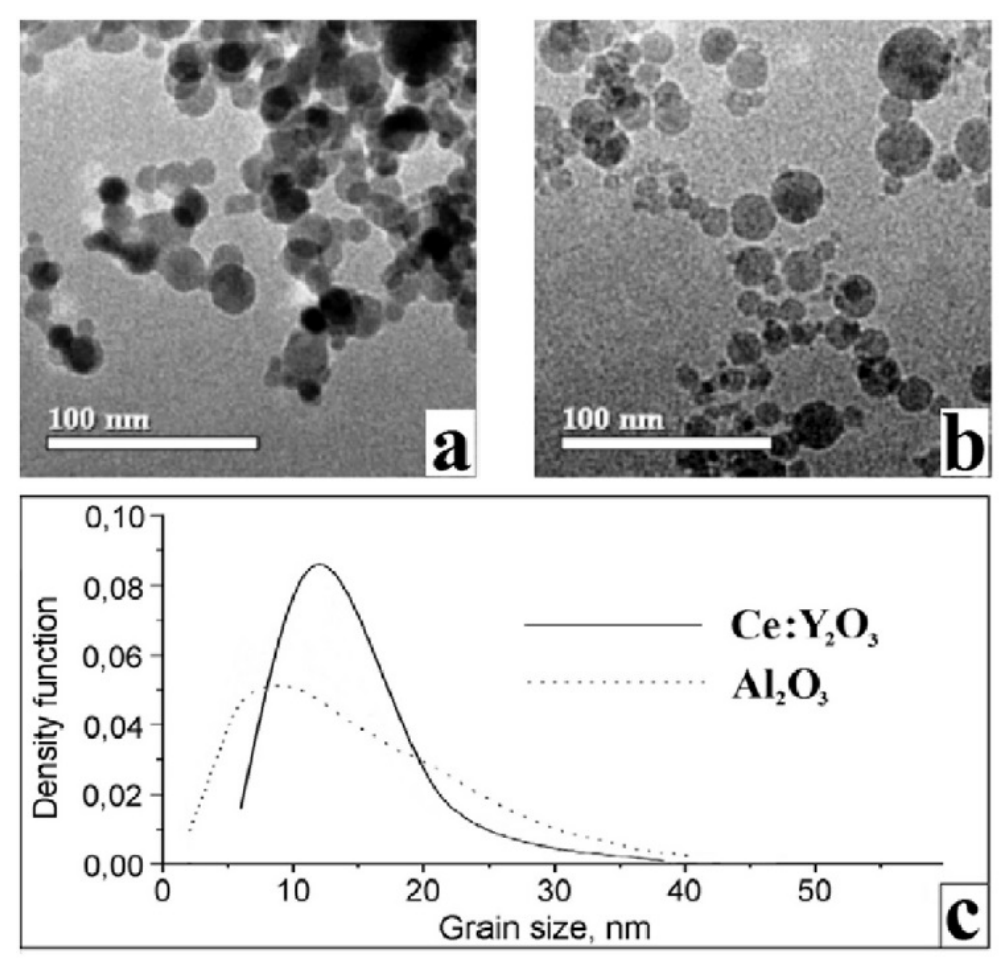

FIG. 1. Transmission electron microscopy (TEM) images of (a) $\mathrm{Ce}: \mathrm{Y}_{2} \mathrm{O}_{3}$ and (b) $\mathrm{Al}_{2} \mathrm{O}_{3}$ nanopowders produced by the laser ablation method. (c) Size distribution of the nanopowders 
destruction of ceramic sample during sintering, this phase transformation was performed in advance by annealing of $\mathrm{Ce}^{3+}: \mathrm{Y}_{2} \mathrm{O}_{3}$ nanopowder at $1000{ }^{\circ} \mathrm{C}$ for $30 \mathrm{~min}$. XRD analysis of annealed nanopowder on D8 Discover diffractometer has shown that content of the cubic phase at these annealing conditions is equal to $100 \%$. According to the BET analysis, the specific surface area of the $\mathrm{Ce}^{3+}: \mathrm{Y}_{2} \mathrm{O}_{3}$ particles after annealing was $18.12 \mathrm{~m}^{2} / \mathrm{g}$, thus showing that the average particle size increased from 11 to $71 \mathrm{~nm}$.

The laser method also was used for producing of $\mathrm{Al}_{2} \mathrm{O}_{3}$ nanopowder. Fig. 1(c) shows the size distribution of $\mathrm{Al}_{2} \mathrm{O}_{3}$ nanopowder (Fig. 1(b)) after sedimentation. Particles are weakly agglomerated and have a weakly faceted near-spherical shape. The distribution peak is realized at $13 \mathrm{~nm}$, the average particle sizes $13 \mathrm{~nm}$. According to the BET analysis, the specific surface area of $\mathrm{Al}_{2} \mathrm{O}_{3}$ nanopowder was $82.96 \mathrm{~m}^{2} / \mathrm{g}$. XRD analysis on D8 Discover diffractometer showed that the content of main $\gamma-\mathrm{Al}_{2} \mathrm{O}_{3}$ crystalline phase is 90 wt.\%. Also, there is a low-temperature $\delta$ - $\mathrm{Al}_{2} \mathrm{O}_{3}$ phase with a content of 10 wt.\%. $\mathrm{Al}_{2} \mathrm{O}_{3}$ nanopowder has been annealed in air at $1200{ }^{\circ} \mathrm{C}$ for $3 \mathrm{~h}$ to receive one phase $\alpha-\mathrm{Al}_{2} \mathrm{O}_{3}$. XRD analysis of annealed nanopowder on D8 Discover diffractometer has shown that content of the $\alpha-\mathrm{Al}_{2} \mathrm{O}_{3}$ phase at these annealing conditions is equal to $100 \%$. According to the BET analysis the specific surface area of the $\mathrm{Ce}^{3+}: \mathrm{Y}_{2} \mathrm{O}_{3}$ particles after annealing was $10.05 \mathrm{~m}^{2} / \mathrm{g}$.

Nanopowders (annealed $\mathrm{Al}_{2} \mathrm{O}_{3}$ and $\mathrm{Ce}: \mathrm{Y}_{2} \mathrm{O}_{3}$ ) were weighed according to the chemical stoichiometry composition of $0.1,1,3,5$ at.\% $\mathrm{Ce}^{3+}: \mathrm{YAG}((\mathrm{Ce}+\mathrm{Y}): \mathrm{Al}=3: 5)$ and mixed with ethyl alcohol and 0.5 mas.\% sintering aid tetraethyl orthosilicate (TEOS). The mass ratio of nanopowder, balls and ethanol was $1: 4: 8$, respectively. Then, the mixed slurry was ball milled in a plastic bottle with YSZ balls for $48 \mathrm{~h}$. After ball milling the slurry was dried using the vacuum rotary evaporator and then annealed at $600{ }^{\circ} \mathrm{C}$ for $3 \mathrm{~h}$ to remove organic impurities.

The compact was prepared from the powders with $\mathrm{Ce}^{3+}$ :YAG crystal structure. For preparation of $\mathrm{Ce}^{3+}: \mathrm{YAG}$ powder, the mixture was compacted into pellets with relative density of $20 \%$. Then, the pellets were annealed in air at $1200{ }^{\circ} \mathrm{C}$ for $3 \mathrm{~h}$. XRD analysis on D8 Discover diffractometer showed that the content of YAG phase in the pellets was 96-98\%. Additionally, the pellets were ball milled in ethyl alcohol with YSZ balls for $48 \mathrm{~h}$. The received powder was uniaxially pressed into disks with a diameter of $15 \mathrm{~mm}$ and a thickness of $2-4 \mathrm{~mm}$ at $200 \mathrm{MPa}$. The density of green body was $2.2 \mathrm{~g} / \mathrm{cm}^{3}$ (relative density of $48 \%$ ).

Then compact was annealed in air at $800{ }^{\circ} \mathrm{C}$ for $3 \mathrm{~h}$ to remove the organic impurities. The compacts were sintered at $1780{ }^{\circ} \mathrm{C}$ for $20 \mathrm{~h}$ in a high temperature vacuum furnace under $3 \cdot 10^{-5}$ mbar vacuum during holding and then annealed at $1300{ }^{\circ} \mathrm{C}$ for $5 \mathrm{~h}$ in air. The as-synthesized samples of transparent Ce:YAG were $10 \mathrm{~mm}$ in diameter and $2 \mathrm{~mm}$ in thickness and are presented in Fig. 2.

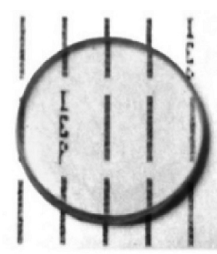

$0.1 \%$ Ce:YAG

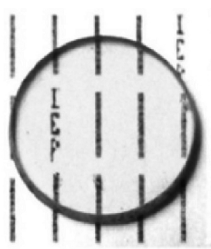

$1 \%$ Ce:YAG

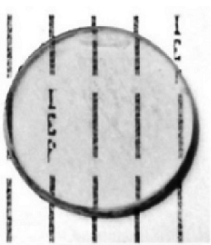

$3 \% \mathrm{Ce}: Y A G$

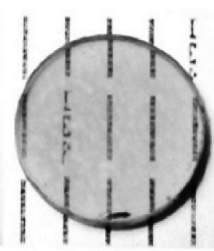

$5 \%$ Ce:YAG

FIG. 2. Photograph of as-synthesized Ce:YAG ceramics $(\varnothing 10 \times 2 \mathrm{~mm})$

The microstructural properties of ceramic Ce:YAG samples were measured using optical microscope OLYMPUS BX51TRF-5.

The optical transmittance and absorption spectra of the obtained Ce:YAG ceramics were measured using a Shimadzu UV-2450 spectrophotometer in the 200-900 nm wavelength range. The X-ray luminescence (XRL) spectra and decay curves were measured using a setup ASNI-ROSTT (experimental physics department, UrFU) on the basis of an MDR-2 monochromator. The XRL channel is equipped by an URS-1 X-ray source $(40 \mathrm{kV}, 10 \mathrm{~mA}$, $\mathrm{W}$-anode) and a Hamamatsu R928 photomultiplier (180-900 nm). The measurements of decay curves were carried out using a pulse electron gun (pulse duration $15 \mathrm{~ns}$, energy $150 \mathrm{keV}$ ), FEU-39 photomultiplier and Tektronix TDS5034B oscilloscope with a 50 Ohm load.

The scintillation properties of ceramic Ce:YAG samples were measured using a "Spectr-1" certified setup equipped by an FEU-130 photomultiplier, spectrometer and spectrometric amplifier "Aspect". The ${ }^{137} \mathrm{Cs}$ and ${ }^{239} \mathrm{Pu}$ were used as $\gamma$ - and $\alpha$-source. A CsI-Tl single crystal with $\varnothing 10 \times 2 \mathrm{~mm}$ size was used as a reference scintillator.

\section{Results and discussion}

Figure 2 shows the photograph of the sintered ceramics obtained after annealing and polishing. The samples appear transparent for all doping concentrations and have a yellow color which is conventional for such material. 
Figure 3 shows the photograph of surfaces of a double polished Ce:YAG ceramics samples after annealing at $1350{ }^{\circ} \mathrm{C}$ for $5 \mathrm{~h}$ and thermal etching. The average grain size is not dependent upon the $\mathrm{Ce}^{3+}$ dopant concentration and is $17 \mu \mathrm{m}$. There is scattering centers of various sizes.

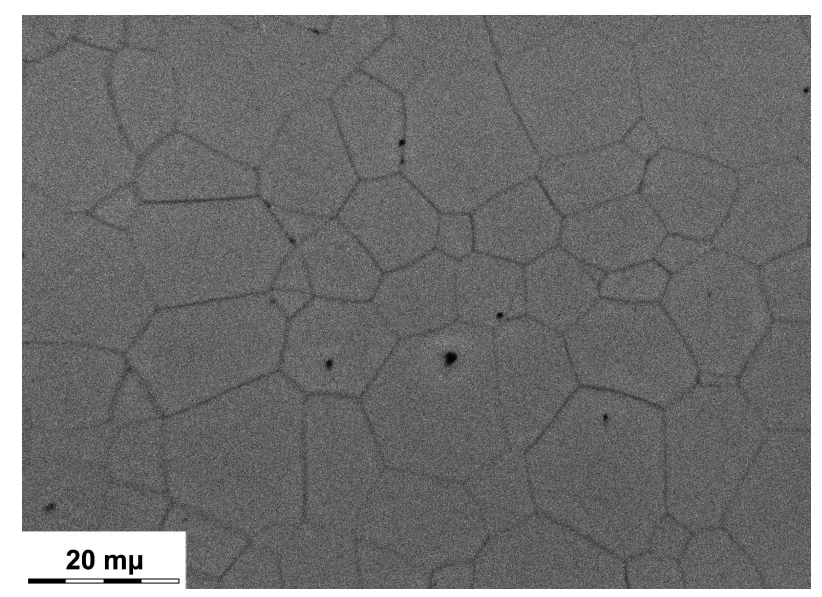

FIG. 3. The photograph of surfaces of a double polished $\mathrm{Ce}^{3+}: \mathrm{YAG}$ ceramics samples

The distribution of the scattering centers at various depths throughout the sample was calculated using optical microscope OLYMPUS. The central area of the samples was scanned at intervals of $50 \mu \mathrm{m}$ with the volume of a sample under analysis being $577 \times 433 \times 50 \mu \mathrm{m}^{3}$. We failed to separate pores from secondary phases, so they were summarized.

The scattering centers were conditionally divided into large - more than $2 \mu \mathrm{m}$ and small - less than $2 \mu \mathrm{m}$. The dimensions of large formations were calculated specifically for each one. As the small scattering centers were usually spherical, therefore for calculation of porosity the average volume of such centers was multiplied by quantity.

The distribution of scattering centers was comparably homogeneous. In some layers, there were large scattering centers with a diameter of more than $10 \mu \mathrm{m}$ that led to significant differences between the concentrations of scattering centers for two nearby layers.

The content of the secondary phases and concentration of pores was $34,74,53,50 \mathrm{ppm}$ for $0.1,1,3$ and $5 \%$ of $\mathrm{Ce}^{3+}$, respectively.

The transmission spectra of 2-mm-thick Ce:YAG ceramics are shown in Fig. 4. As can be seen from the figure the highest transmittance of over $81 \%$ in the range of 500-900 nm has the sample with the lowest doping level of $\mathrm{Ce}^{3+}\left(0.1\right.$ at.\%). The optical transmittance of samples depends on the $\mathrm{Ce}^{3+}$ dopant concentration and is equal to 72 and $77 \%$ for $1 \%$ and for $3 \%$ and $5 \%$ of $\mathrm{Ce}^{3+}$, respectively. In the transmission and absorption spectra $4 f \rightarrow 5 d^{1}$ and $4 f \rightarrow 5 d^{2}$ absorption bands of trivalent cerium are observed at 337 and $457 \mathrm{~nm}$. With an increase in the $\mathrm{Ce}^{3+}$ content, the absorption bands become stronger and the edge of the transparent region near $500 \mathrm{~nm}$ is shifted to the right side. The change of the edge position leads to a color modification of samples (Fig. 3). The bands located below $340 \mathrm{~nm}$ with the maxima at 225,258 and $301 \mathrm{~nm}$ can be attributed to absorption transitions in $\mathrm{Ce}^{4+}$-centers. A similar fine structure of absorption bands in Ce:LuAG and a description of $\mathrm{Ce}^{4+}$-centers formation in a garnet host was reported in [22].

The XRL measurements of the fabricated ceramic samples show that the bright $d-f$ luminescence of $\mathrm{Ce}^{3+}$ is observed in the 425-700 nm region with a maximum at 527-545 nm. The XRL spectra consist of two luminescence bands attributed to ${ }^{2} \mathrm{D} \rightarrow{ }^{2} \mathrm{~F}_{7 / 2}$, and ${ }^{2} \mathrm{D} \rightarrow{ }^{2} \mathrm{~F}_{5 / 2}$ transitions in $\mathrm{Ce}^{3+}$ ions [23]. The maximum intensity of luminescence has the sample with 1 at.\% of Ce. An increase in the cerium concentration from 1 to 3 and 5 at.\% leads to concentration quenching of luminescence and the intensity of XRL is reduced (Fig. 5). For various Ce concentrations, the positions of maxima are changed due to the variation of the absorption edge near 470-520 nm (Fig. 4).

Besides the $\mathrm{Ce}^{3+}$ luminescence bands, the luminescence of self-trapped excision (STE) in YAG [24] is observed in the 250-450 nm region (Fig. 5). The shape of the STE band is due to the shape of $\mathrm{Ce}^{3+}$ absorption bands (see Fig. 4(b)). The intensity of the STE band decreases with an increase in dopant concentration. The STE band is almost completely absent in samples doped with 3 and $5 \%$ of Ce. The observed narrow band at $312 \mathrm{~nm}$ (Fig. 5) is attributed to a well-known $f-f$ transition in uncontrolled $\mathrm{Gd}^{3+}$ impurity ions. 


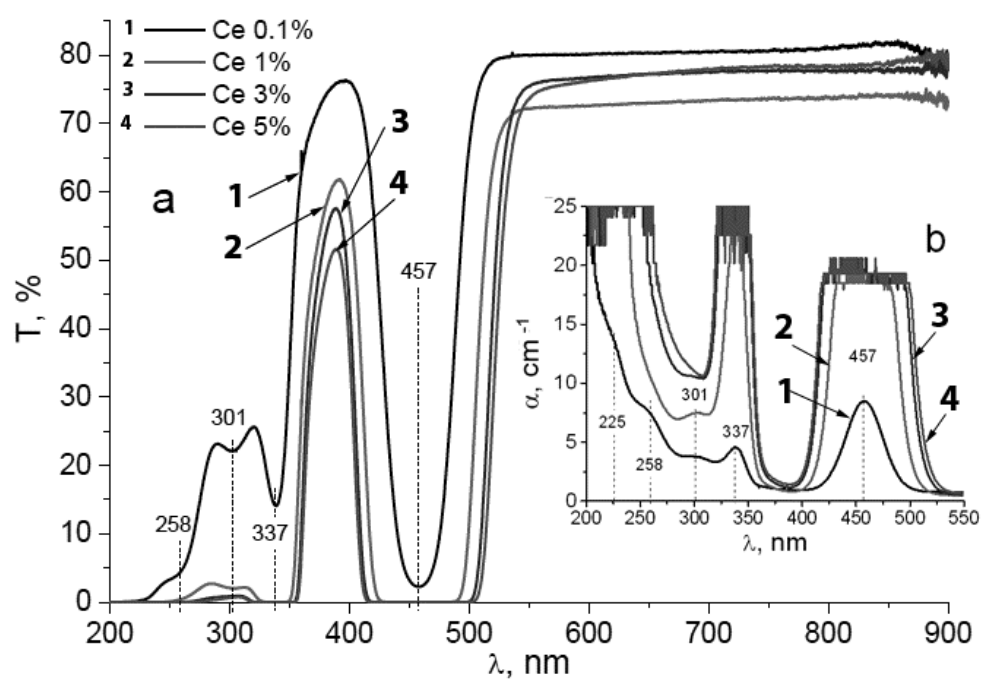

FIG. 4. The optical transmittance (a) and absorption (b) spectra of ceramic Ce:YAG samples

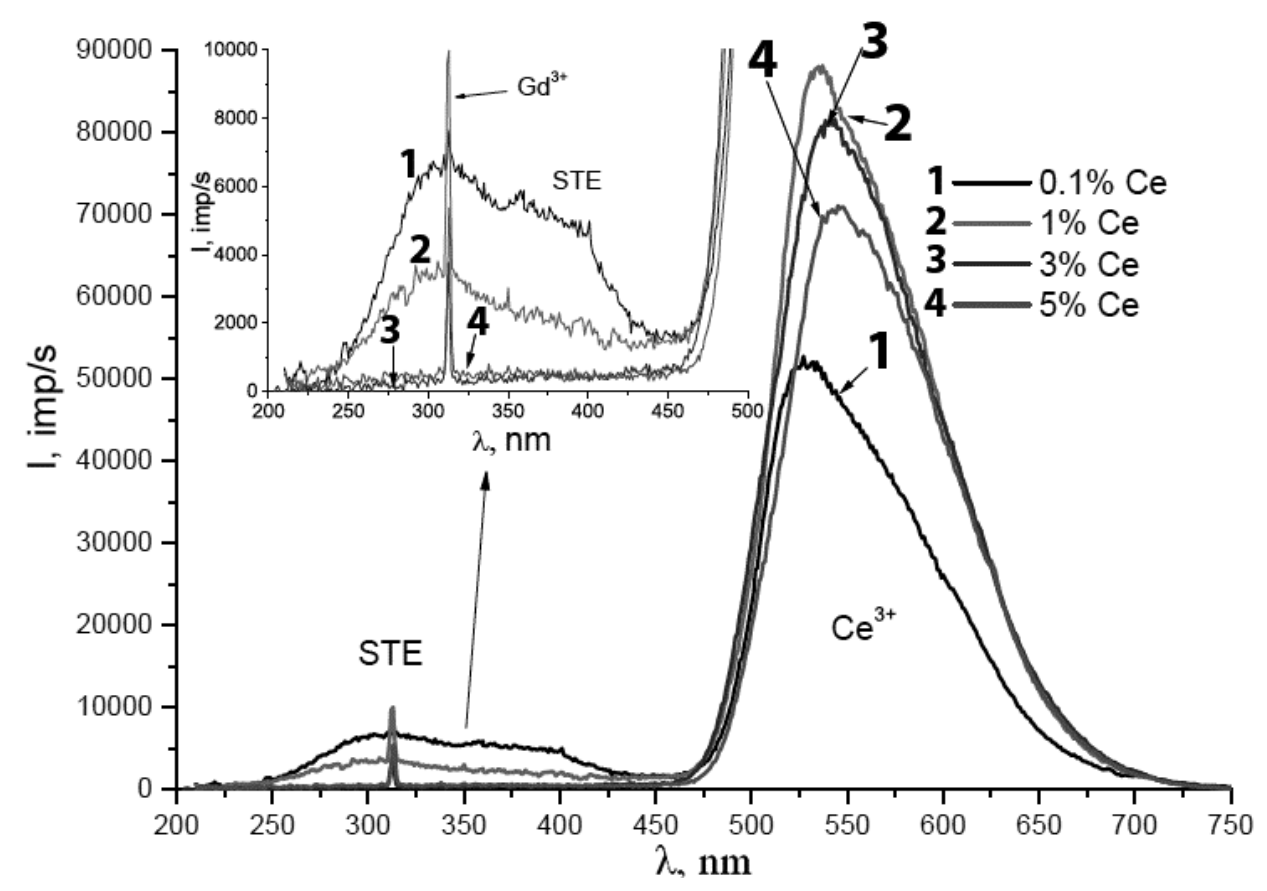

FIG. 5. The XRL spectra of ceramic Ce:YAG samples

For a detailed study of energy relaxation processes in Ce:YAG ceramics, the decay curves (Fig. 6) under a pulse electron beam were measured. The decay parameters (Table 1) were measured in the $545 \mathrm{~nm}$ band (for $0.1 \%$ Ce also in $390 \mathrm{~nm}$ ) and identified through the fitting procedure which was performed using the expression with the sum of two exponents, $I(t)=A_{1} e^{-t / \tau_{1}}+A_{2} e^{-t / \tau_{21}}$, where $A_{i}$ - intensity of component, $\tau_{i}-$ decay constant of the decay curve.

The decay curve measured in the STE band at $390 \mathrm{~nm}$ had fast and slow components (Fig. 6, the decay parameters for the STE band are presented in Table 1). The fast component was attributed to a relaxation of a convenient STE which was localized at Al-sites. The slow component can be attributed to the relaxation of the defect-bound exciton (DBE). The $\mathrm{Y}^{3+} \mathrm{Al}$ antisite defects are likely possible defects for the DBE creation in the YAG host [24].

The decay curve measured in the $\mathrm{Ce}^{3+}$ band at $545 \mathrm{~nm}$ also had two components (Fig. 6). Table 1 shows that with an increase in the $\mathrm{Ce}^{3+}$ concentration, the decay constants decreased from 110 to $26 \mathrm{~ns}$ for the fast component 


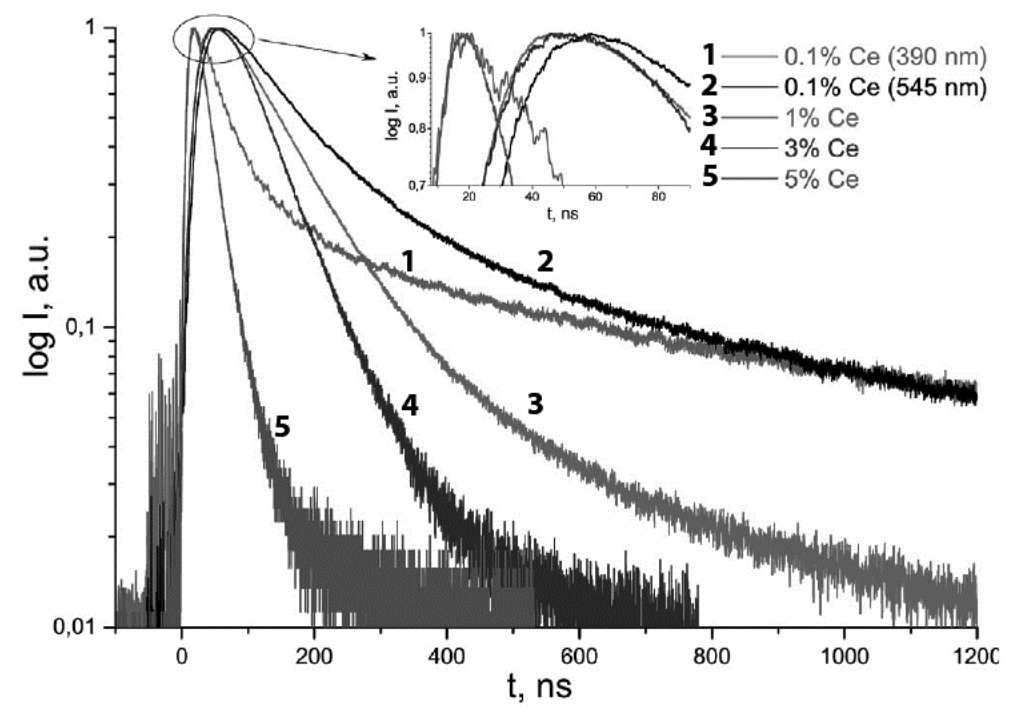

FIG. 6. The decay curves of ceramic Ce:YAG samples under pulse cathode beam collected at $545 \mathrm{~nm}$ wavelength

TABLE 1. The decay parameters of ceramic Ce:YAG pulse cathode luminescence

\begin{tabular}{|c|c|c|c|c|c|c|}
\hline Sample & $\tau_{1}, \mathrm{~ns}$ & $A_{1}, \%$ & $\tau_{2}, \mathrm{~ns}$ & $A_{2}, \%$ & Reduced Chi-Sqr & Adj. R-Square \\
\hline $0.1 \% \mathrm{Ce}(390 \mathrm{~nm})$ & 45.9 & 87 & 541 & 13 & $1.61 \cdot 10^{-5}$ & 0.99823 \\
\hline $0.1 \% \mathrm{Ce}(545 \mathrm{~nm})$ & 110 & 85 & 562 & 15 & $5.53 \cdot 10^{-6}$ & 0.99972 \\
\hline $1 \% \mathrm{Ce}$ & 99 & 96 & 526 & 4 & $3.04 \cdot 10^{-6}$ & 0.9998 \\
\hline $3 \% \mathrm{Ce}$ & 72 & 99.5 & 489 & 0.5 & $9.65 \cdot 10^{-6}$ & 0.99958 \\
\hline $5 \% \mathrm{Ce}$ & 26 & 99.0 & 338 & 1.0 & $1.26 \cdot 10^{-5}$ & 0.99915 \\
\hline
\end{tabular}

and from 562 to $338 \mathrm{~ns}$ for the slow component. The relative intensity ( $A_{1}$ in Table 1 ) of the fast component increases with an increase in the $\mathrm{Ce}^{3+}$ concentration. However, the intensity of the slow component in the decay curve of highly doped ( 3 and $5 \%$ ) samples was negligible and practically the entire part of the $\mathrm{Ce}^{3+}$ luminescence had a fast decay time.

The fast component of the decay curve for the sample doped with $5 \%$ of $\mathrm{Ce}^{3+}$ is $26 \mathrm{~ns}$. A similar value of decay time $(\sim 28 \mathrm{~ns})$ was reported for the luminescence decay of YAG nanoparticles doped with 4 at.\% of Ce [25]. The short decay time for the luminescence of $\mathrm{Ce}^{3+}$ ions in YAG host is unusually low and can be connected with a concentration quenching effect. The decay time variation of the luminescence for the $\mathrm{Ce}^{3+}$ band in YAG for various dopant concentrations was also presented in [26].

The slow decay component of $\mathrm{Ce}^{3+}$ luminescence is usually connected with the involvement in the relaxation processes of point defects such as oxygen vacancies, which can be shallow traps for charge carriers. The presence of such shallow traps significantly increases the decay time of luminescence due to the absorption and reabsorption of charge carriers. The decrease of decay time and the intensity of the slow component with an increase in the $\mathrm{Ce}^{3+}$ concentration indicates the reduction of or/and transformation of point defects in the YAG lattice. This explanation also seems plausible since the decay curves of the samples doped with $0.1,1$ and 3 at. $\%$ of $\mathrm{Ce}^{3+}$ have a region of increase (see inset in Fig. 6). The durations of this increased region in the decay curves decrease with an increase in Ce concentration. The presence of the region of increase in the luminescence decay curve usually indicates the intermediate localization of the charge carriers in the shallow traps and reflects the migration processes of electronic excitations in the crystal lattice. However, for the sample doped with 5 at. $\%$ of $\mathrm{Ce}^{3+}$, the region of increase for the decay curve is absent (Fig. 6). Conversely, the observed behavior of the decay parameters of the $\mathrm{Ce}^{3+}$-center luminescence (for the fast and slow components) appears to be an influence of the concentration quenching effect on relaxation processes. In addition, the content of $\mathrm{Ce}^{4+}$-ions in the YAG lattice can affect the efficiency of non-radiative relaxation by the $\mathrm{Ce}^{3+} \rightarrow \mathrm{Ce}^{4+}$ interaction channel. The origin of the very fast luminescence decay of $\mathrm{Ce}^{3+}$ in highly doped YAG needs further study. 
In order to determine the scintillation properties of the obtained Ce:YAG ceramics, the pulse-height spectra (PHS) were measured under $\alpha$ - and $\gamma$-source excitations. The pulse height spectra are presented in Figs. 7 and 8. The main parameters of ceramic Ce:YAG samples are presented in Table 2. The data for YAG ceramic and single crystal is also presented for comparison. A commercial CsI:Tl scintillator with the same sizes is used as a reference sample. ALY was calculated using a CsI:Tl single crystal. As can be seen from Table 2, YAG doped with 1 at.\% of $\mathrm{Ce}^{3+}$ has a the highest absolute light yield and the lowest $\alpha / \gamma$ ratio among the other investigated Ce:YAG samples. Energy resolutions of the fabricated ceramic scintillator are not high (10-15\%) due to a low effective atomic number. The resolution can be increased using thicker samples.

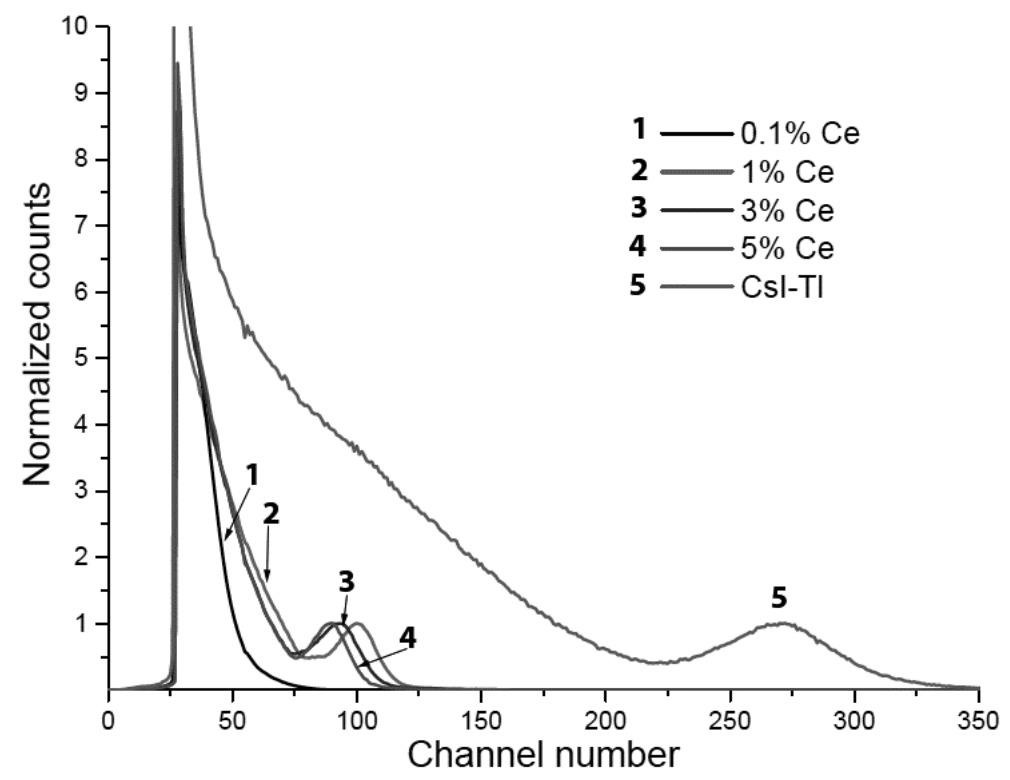

FIG. 7. gamma-ray pulse-height spectra of ${ }^{137} \mathrm{Cs}$ measured using the ceramic Ce:YAG samples with varied concentration of $\mathrm{Ce}$ in comparison with standard CsI:Tl scintillator $(\varnothing 10 \times 2 \mathrm{~mm})$

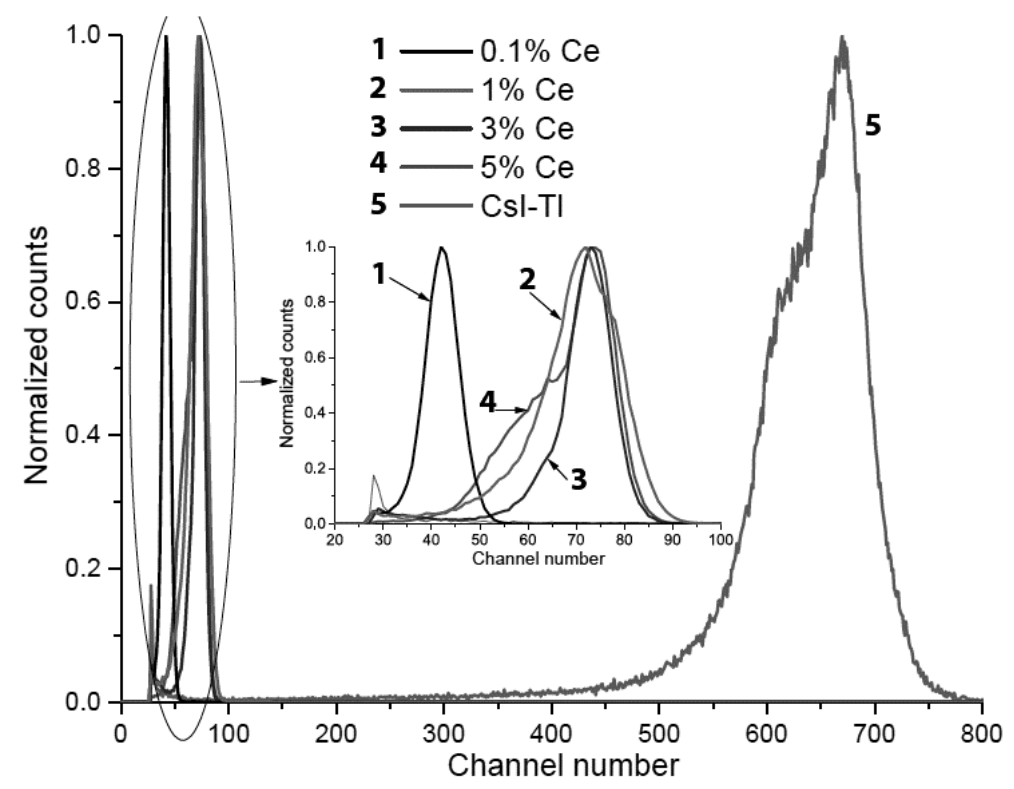

FIG. 8. The alpha particles pulse-height spectra of ${ }^{239} \mathrm{Pu}$ measured using the ceramic Ce:YAG samples with varied concentration of $\mathrm{Ce}$ in comparison with standard CsI:Tl scintillator $(\varnothing 10 \times$ $2 \mathrm{~mm})$ 
TABLE 2. The scintillation properties of ceramic Ce:YAG samples

\begin{tabular}{|c|c|c|c|c|}
\hline \multirow{2}{*}{ Sample } & \multicolumn{2}{|c|}{$\gamma$-ray $\left({ }^{137} \mathrm{Cs}, 662 \mathrm{keV}\right)$} & $\begin{array}{c}\alpha \text {-particles } \\
\left({ }^{239} \mathrm{Pu}, 5.15 \mathrm{MeV}\right)\end{array}$ & \multirow{2}{*}{$\alpha / \gamma$ ratio } \\
\cline { 2 - 5 } & $\begin{array}{c}\text { Relative light } \\
\text { yield, } \%\end{array}$ & $\begin{array}{c}\text { Absolute light } \\
\text { yield, ph/MeV }\end{array}$ & $\begin{array}{c}\text { Relative light } \\
\text { yield, } \%\end{array}$ & \\
\hline CsI:Tl & 100 & 65000 & 100 & - \\
\hline $0.1 \% \mathrm{Ce}$ & $7.6^{*}$ & $4940^{*}$ & 3.2 & 0.055 \\
\hline $1 \% \mathrm{Ce}$ & 32 & 20800 & 7.8 & 0.032 \\
\hline $3 \% \mathrm{Ce}$ & 29.2 & 18980 & 8.0 & 0.036 \\
\hline $5 \% \mathrm{Ce}$ & 27.6 & 17940 & 8.1 & - \\
\hline $\begin{array}{c}0.27 \% \mathrm{Ce}: Y A G \\
\text { single crystal [27] }\end{array}$ & - & 19802 & - & - \\
\hline $\begin{array}{c}0.5 \% \mathrm{Ce}: Y A G \\
\text { ceramic }[28]\end{array}$ & 37 & - & - & - \\
\hline
\end{tabular}

*Parameter is obtained from the evaluation due to the absence of photopeak.

\section{Conclusions}

Nanopowders of $\mathrm{Ce}_{2 x} \mathrm{Y}_{2-2 x} \mathrm{O}_{3}\left(x=0.001,0.01,0.03\right.$, and 0.05) and $\mathrm{Al}_{2} \mathrm{O}_{3}$ synthesized by laser ablation were used to fabricate transparent Ce:YAG ceramics via a solid-state reaction method with an additional round of pre-calcining. The microstructural, optical, radioluminescence and scintillation properties of the obtained ceramics were investigated. The content of the secondary phases and pore concentration in most transparent $(81 \%$ in the range of 500-900 nm) Ce:YAG ceramic sample with $0.1 \%$ of $\mathrm{Ce}^{3+}$ was $34 \mathrm{ppm}$. The average grain size did not depend on the $\mathrm{Ce}^{3+}$ dopant concentration and was $17 \mu \mathrm{m}$. The Ce:YAG ceramic sample with $1 \%$ of $\mathrm{Ce}^{3+}$ gave the most intense luminescence and highest absolute and relative light yields under $\alpha$ and $\gamma$ ionizing radiation. However, the Ce:YAG ceramic sample doped with $5 \%$ of $\mathrm{Ce}^{3+}$ gave the best kinetic characteristics. The $5 \%$ Ce:YAG has the main fast component with a 26 ns decay time and a negligible weak slow component with a decay time of 338 ns. Herewith, the ALY was slightly less than that of $1 \%$ Ce:YAG.

A ceramic Ce:YAG scintillator can be attractive for positron emission tomography (PET) and similar devices owing to the applicability for any system with photodiode registration and short decay times.

\section{Acknowledgements}

The work was fulfilled in the frame of state task project No. 0389-2014-0027 (2016-2017) and supported by Act 211 Government of the Russian Federation, contract No. 02.A03.21.0006.

\section{References}

[1] Borisevich A. et al. On the development of heavy and fast scintillation nano-ceramics. IEEE Nulcear Science Symposium Conference Record, Dresden, Germany, 19-25 October 2008, P. 3533-3535.

[2] Greskovich C., Duclos S. Ceramic scintillators. Annu. Rev. Mater. Sci., 1997, 27 (1), P. 69-88.

[3] Ikesue A., et al. Ceramic lasers. Cambridge University Press, Cambridge, 2013, 444 .

[4] Deshmukh P., Satapathy S., Singh M.K., Gupta P.K. Effect of surfactant concentration and solvent used for washing in the preparation of $\mathrm{Yb}: \mathrm{Y}_{2} \mathrm{O}_{3}$ transparent ceramics. Nanosystems: physics, chemistry, mathematics, 2016, 7 (3), P. $534-537$.

[5] Nikl M., Yanagida T., et al. Optical Ceramics for Fast Scintillator Materials. Recent Advances in Ceramic Materials Research, Nova Science Publishers, Inc., 2013, P. 127-176.

[6] Kaminskii A.A. Laser crystals and ceramics: recent advances. Laser Photon Rev., 2007, 1 (2), P. 93.

[7] Mihokova E., Nikl M., et al. Luminescence and scintillation properties of YAG:Ce single crystal and optical ceramics. J. Lumin., 2007, 126, P. 77-80.

[8] Bok J., et al. Effect of oxidation annealing on optical properties of YAG:Ce single crystals. Opt. Mater., 2015, 46, P. 591-595.

[9] Gong M., et al. Novel synthesis and optical characterization of phosphor-converted WLED employing Ce:YAG-doped glass. J. Alloy. and Comp., 2016, 664, P. 125-132.

[10] Pricha I., et al. Layered Ceramic Phosphors Based on CaAlSiN 3 :Eu and YAG:Ce for White Light-Emitting Diodes. J. Am. Ceram. Soc., 2016, 99 (1), P. 211-217.

[11] Liu G.H., et al. Ce:YAG transparent ceramics for applications of high power LEDs: Thickness effects and high temperature performance. Mater. Lett., 2015, 139, P. 480-482.

[12] Ikesue A.J. Ce:YAG Ceramic Scintillator for Electron Beam Detector. Ceram. Soc. Japan, 2000,108 (11), P. 1020-1023.

[13] Kochawattana S., Stevenson A., et al. Sintering and grain growth in $\mathrm{SiO}_{2}$ doped Nd:YAG. J. Eur. Ceram. Soc., 2008,28 (7), P. 1527-1534. 
[14] Byer R.L. Progress in engineering ceramics for advanced solid-state lasers. 3rd Laser Ceramics Symposium, Paris, France, 8-10 October 2007.

[15] Lu J., Ueda K., et al. Doped and undoped yttrium aluminium garnet $\left(\mathrm{Y}_{3} \mathrm{Al}_{5} \mathrm{O}_{12}\right)$ nanocrystalline ceramics - a new generation of solid state laser and optical materials. Compound, 2002, 341, P. 220-225.

[16] Lu J., Yagi H., et al. $110 \mathrm{~W}$ ceramic $\mathrm{Nd}^{3+}: \mathrm{Y}_{3} \mathrm{Al}_{5} \mathrm{O}_{12}$ laser. Appl. Phys. B., 2004, 79 (1), P. $25-28$.

[17] Chung B., Park J., Sim S. Synthesis of yttrium aluminum garnet powder by a citrate gel method. J. Ceram. Proc. Res., 2003 , 4, P. 145.

[18] Chen D., Jordan E.H., Gell M. Dense AluminaZirconia Coatings Using the Solution Precursor Plasma Spray Process. J. Am. Ceram. Soc., 2008, 91, P. 2759-2762.

[19] Ge X., Sun Y., Liu C., Qi Wu. Influence of combustion reagent and microwave drying method on the characteristics of nano-sized $\mathrm{Nd}^{3+}$ :YAG powders synthesized by the gel combustion method. J. SolGel Sci. Technol., 2009, 52, P. 179-187.

[20] Bagayev S.N., Osipov V.V., et al. Fabrication of $\mathrm{Nd}^{3+}$ :YAG laser ceramics with various approaches. Opt. Mater., 2012,34, P. $1482-1487$.

[21] Osipov V.V., Kotov Yu.A., et al. Laser synthesis of nanopowders. Laser Phys., 2006, 16, P. 116-125.

[22] Liu S., et al. Optical, luminescence and scintillation characteristics of non-stoichiometric LuAG:Ce ceramics. J. Lumin., 2016, 169, P. $72-77$.

[23] Blasse G., Bril A. A new phosphor for flyingspot cathoderay tubes for color television: yellowemitting $\mathrm{Y}_{3} \mathrm{Al}_{5} \mathrm{O}_{12} \mathrm{Ce}^{3+} . A_{p p l}$ Phys. Lett., 1967, 11 (2), P. 53.

[24] Babin V., et al. Luminescence of undoped LuAG and YAG crystals. Phys. Stat. Sol. (c), 2005,2 (1), P. $97-100$.

[25] Masenelli B., et al. YAG:Ce nanoparticle lightsources. Nanotechnol., 2013, 24, 165703 (7 p.).

[26] Schauer P. Optimization of decay kinetics of YAG:Ce single crystal scintillators for S(T)EM electron detectors. Nucl. Instr. Methods Phys. Res. B, 2011, 269, P. 2572-2577.

[27] Mares J.A., et al. Scintillation properties of $\operatorname{Pr}^{3+}$-doped lutetium and yttrium aluminum garnets: Comparison with Ce ${ }^{3+}$-doped ones. Opt. Mat., 2011, 34 (2), P. 424-427.

[28] Yanagida T., et al. Evaluation of properties of YAG (Ce) ceramic scintillators. IEEE Trans. Nuc. Sci., $2005, \mathbf{5 2}$ (5), P. 1836-1841. 